\title{
Análisis comparativo de dos metodologías para la asignación de costos en producción de leche bovina. Estudio de casos
}

\author{
Comparative analysis of two methodologies for the assignment \\ of costs in bovine milk production. Study of cases
}

\author{
Juan Lerdon F. ${ }^{1}$, Juan Carlos Miranda C. ${ }^{2}$, Victor Moreira L. ${ }^{1}$, Marcelo Gebauer R. ${ }^{1}$
}

\section{RESUMEN}

Los actuales precios de la leche obligan a controlar los costos y optimizar las decisiones de producción, junto con la necesidad de aumentar las escalas debido a una disminución de los márgenes. Surge entonces la interrogante: ¿cuán diferente es, en el análisis de costo del litro de leche, aplicar distintos métodos de costeo? Esto confiere al trabajo la importancia de ser el primero en analizar diferencias entre métodos de costos, aplicados a la producción de leche bovina. A partir de los antecedentes económicos de 14 predios productores de leche vinculados al Centro de Gestión Agrícola de Paillaco (CEGE Paillaco), y utilizando análisis multivariante, se compararon dos métodos de costos utilizados a nivel nacional: por diferencia residual y por producción equivalente, para determinar si existían diferencias entre ambos. En una primera fase se utilizó una clasificación jerárquica (cluster) de la información entregada por el dendrograma. Luego se realizó la clasificación no jerárquica (K-means), intentando con este método mover los casos de un grupo a otro para conseguir los resultados ANOVA, que son más significativos. Lograda la clasificación, se realizó el análisis discriminante, determinando la contribución de las variables relevantes que clasifican a los agricultores en el grupo al que pertenecen. Respecto de los resultados del análisis económico de los datos, aplicados a ambos métodos de costos, se observan diferencias en el costo del litro de leche de cada agricultor. Del análisis estadístico de los datos y al evaluar la estructura, en la clasificación y calificación de los datos, se observó que no son diferentes estadísticamente; ambos métodos son capaces de discriminar los grupos con alta significancia, $\chi_{(15 ; 0,05)}^{2}(\mathrm{P}<0,01)$.

Palabras clave: producción de leche, asignación de costos, análisis multivariante.

\begin{abstract}
Current milk prices force to control costs and optimize production decisions along with the necessity to increase production scales by margins decreasing. Then at this point emerges the question of how different the application of cost methods is when analyzing milk cost by applying different costing methods?. This question confers to the work the importance of being the first in analyzing differences between cost methods applied to bovine milk production. The study was applied to the economic antecedents of 14 cases, all milk-producer farmers, given by the CEGE Paillaco. The general objective of the study is to analyze two cost methods used at national level: residual difference and equivalent production, to determine differences in results between both methodologies. The study is based in multivariant analysis. In a first stage, a hierarchical classification was used (Cluster), the nonhierarchic classification (K-means) was made from the information given by the Dendrogram. Moving cases from one group to another is carried out by means of this method, so as to obtain ANOVAS results, which are more significant. Once classification was obtained, the discriminant analysis was made to determine the contribution of the relevant variables that classify farmers into the appropriate group each one belongs. The results evidence that the economic analysis of the data applied to cost methods, differences in the results by cost of milk liter of each farmer are observed. From the statistical analysis of the data, and after evaluating the structure of both methods of costing, both methods are observed to be statistically similar concerning data classification and qualification; both methods are able to discriminate the groups with high significance, $\chi 2_{(15 ; 0.05)}(P<0.01)$.
\end{abstract}

Key word: milk production, assignment of costs, multivariant analysis.

\section{Introducción}

La producción de leche representa uno de los rubros más importantes del sector agropecuario del sur del país, el que inserto en una economía globalizada, enfrenta condiciones de mercado complejas, que obligan a visualizar el negocio lechero como una empresa, planificando y controlando, de tal manera

\footnotetext{
1 Universidad Austral de Chile, Facultad de Ciencias Agrarias, Instituto de Economía Agraria. Casilla 567. Valdivia, Chile.

2 Universidad Austral de Chile, Facultad de Ciencias Económicas y Administrativas, Instituto de Estadística. Casilla 567. Valdivia, Chile.
} 
de obtener un resultado financiero positivo, es decir, una utilidad y no una pérdida. En este sentido la estimación de los costos unitarios de producción son relevantes para determinar la eficiencia productiva, así como las economías y deseconomías de escala, situación que se ve incrementada por la disminución de precios y rentabilidad. Esto obliga a controlar los costos, optimizar las decisiones de producción y aumentar las escalas de producción debido a la disminución de los márgenes.

En este contexto, cuando existen costos comunes a varias actividades y no es posible determinar exactamente qué partes del costo total corresponden a cada actividad, surge el problema de distribución o asignación, el que tendrá siempre un cierto grado de arbitrariedad, ya que no es posible determinar el costo atribuible a cada objeto de medición (Leyton y Niño, 1994). Asimismo, al determinar los costos se deben respetar ciertos niveles de asignación obligatorios relacionados con las necesidades de valoración periódica del patrimonio y de los resultados de la empresa (Mallo et al., 2000). Se debe además tener la capacidad y la libertad de construir el razonamiento económico que se adecue a cada empresa (Baker et al., 1992; Horngren et al., 1996 y 2001). Así, existen cuatro métodos generales para la determinación de los costos de una empresa, entregando cada uno de ellos resultados distintos (Frank, 1998a y Mallo et al., 2000): método de división, método de las equivalencias, método de suplemento, y método por diferencia residual. Mayores detalles de cada caso pueden ser revisados en Frank (1998a).

En el caso de la agricultura, y más específicamente en el rubro lechero, dos métodos de costeo se usan en forma habitual: por equivalencias y por diferencia residual. Respecto de la determinación del costo del litro de leche por producción equivalente, este método consiste en dividir el ingreso total de las ventas, tanto del producto principal como de los secundarios, por el precio del producto principal (Frank, 1998a). Para la lechería el resultado sería la suma de los ingresos de todos los productos derivados de la producción de leche, divididos por el precio de la leche, denominándose lo anterior producción equivalente. Este método tiene como restricción no generar resultados satisfactorios cuando los ingresos secundarios de otros rubros participan por sobre el $30 \%$ del ingreso total (crianza, engorda, venta de granos, tubérculos) (Frank, 1998b). En el cálculo del costo del litro de leche por diferencia residual la determinación del ingreso del rubro lechería se hace considerando exclusivamente la producción de leche, las ventas de vacas de desecho, la producción de terneros y la diferencia de inventario. Se excluye la venta de vaquillas, por considerarse una actividad independiente a la lechería (Lerdon et al., 1984). En la actualidad no existe consenso en qué sistema de costeo es el más apropiado para el caso de una lechería, es por ello que el objetivo del presente trabajo es comparar y analizar el método de estimación del costo del litro de leche por producción equivalente y por diferencia residual, para determinar si existen diferencias entre ambos.

\section{Material y Método}

\section{Datos y análisis estadístico}

Este trabajo está basado en la información contable del período 2001, de 14 predios productores de leche asociados al Centro de Gestión de Paillaco (CEGE Paillaco). En términos de superficie, abarcan 633 ha, de las cuales 513 ha son destinadas directamente a la lechería. Además poseen una masa ganadera de 404 animales, cuya producción anual es de 1.255.447 L de leche. Se utilizaron planillas electrónicas para la determinación de los costos de producción, aplicando para ello dos métodos de cálculo: diferencia residual, explicado por Lerdon et al. (1984) y producción equivalente, explicado por Frank (1998b).

Para el análisis estadístico de los datos se utilizó el programa SPSS versión 10.0, aplicándose métodos multivariantes que permiten, a partir de un conjunto de datos con información relevante, acerca de una serie de casos (productores), organizarlos en grupos homogéneos a base de la información de variables independientes (variables de costo del litro de leche), provenientes de cada método de costeo. En la clasificación, en una primera fase, se aplicó el método de cluster a una selección de variables de costo de litro de leche, utilizando solo aquellas que caracterizan los objetos que se agrupan y que se refieren específicamente a los objetivos del análisis de cluster, ya que la técnica no diferencia entre variables relevantes e irrelevantes. Este método de clasificación jerárquica cluster no necesita de una definición previa del número de grupos para determinar el número idóneo de grupos (Díaz de Rada, 1998; Pérez, 2001; Hair et al., 1999, Johnsons, 
2000). Posteriormente se realizó una segunda fase de clasificación no jerárquica (K-means), donde se debe proponer el número de grupos, que se obtiene de la primera fase de análisis. Para el estudio de proximidades entre los distintos agricultores se utilizó la distancia euclidiana, distancia que es sensible a la diferencia de escalas entre las variables, ya que aquellas con mayor dispersión tendrán más impacto en el valor de similaridad. Por esta razón, se opta por estandarizar las variables por la medida $Z$ (media cero y desviación típica 1), para eliminar el sesgo que se introduce por las diferencias de escalas. Para la unión del cluster se especifica el método de agregación de Ward, o enlace de mínima varianza, que considera como distancia entre grupos el menor incremento de varianza residual global. La pareja de grupos que produzca el mínimo incremento en esta varianza residual será la elegida para su unión en un nuevo nivel, minimizando la suma de los cuadrados de los residuos entre cluster (Pérez, 2001). La clasificación no jerárquica (K-means) se realizó a partir de la información proporcionada por el dendrograma resultado del cluster jerárquico, donde se considera como un análisis de la varianza, pero el test de significación para la hipótesis de las medias de los grupos es diferente en ANOVA, el que evalúa la variabilidad entre grupos sobre la variabilidad intragrupos (Gondar, 2003; Pérez, 2001). Con este criterio se intenta mover los casos de un grupo a otro para conseguir los resultados ANOVA más significativos. Una vez obtenida la clasificación, se realizó un análisis discriminante con fines de evaluación y explicación. Este análisis permite la validación estadística de los resultados obtenidos, evaluación de las agrupaciones y explicación de las variables relevantes en la discriminación entre grupos. Determina la contribución de cada variable clasificadora a la clasificación correcta, es decir, determina a cada uno de los grupos como al grupo al que pertenecería cada caso, para el que se conocen los valores de las variables (Levy, 2003; Hair et al., 1999).

\section{Cálculo de costos totales}

Las variables de costos que considera el CEGE Paillaco en el desarrollo de la información de costos de sus asociados, que constituyen la base del presente análisis estadístico, se presentan en el Tabla 1.

Para el método de costeo por diferencia residual (MCDR) se consideraron las variables calculadas a valor de costo por litro de leche. Para esto, a la variable $X_{i}$ se le descuentan los ingresos de los subproductos (venta vaca de desecho, terneros y diferencia de inventario), según la importancia porcentual de la variable $X_{i}$ de los costos totales y estos se dividen por el total de litros producidos, como se expresa en la siguiente fórmula:

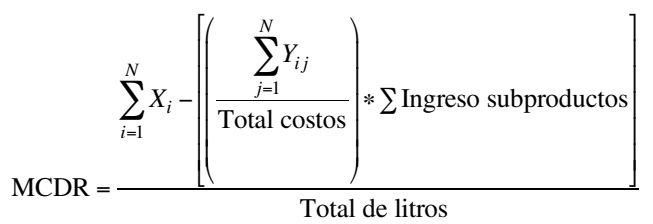

Para el método de costo de producción equivalente (MCPE) se consideraron las variables calculadas a valor de costo por litro de leche. Para esto la variable $X_{a 1}$ se divide por el cuociente entre el total de los ingresos (venta de leche, vacas desecho, terneros y diferencia de inventario) y el precio promedio pagado por litro de leche, como se expresa en la siguiente fórmula:

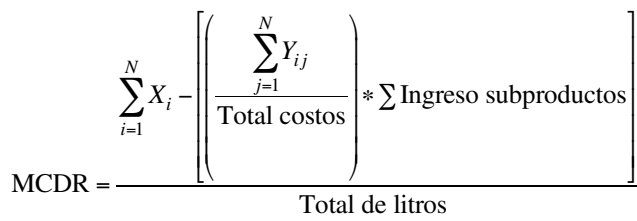

Valoración de activos. La valoración de los activos se realizó de la siguiente manera:

- Suelo agrícola: valor comercial, que es el precio existente en el mercado de acuerdo con la calidad del suelo, ubicación y accesos, entre otros. Este valor no considera la depreciación, al ser el suelo un activo no depreciable.

- Construcciones, instalaciones, maquinarias y equipos: se valoraron por el precio consignado en documentos que acrediten su construcción, debidamente actualizados y depreciados a la fecha del inventario. En caso de no contar con dichos documentos se valorizaron efectuando una estimación de su valor a la fecha del inventario, considerando el costo directo de la inversión y el estado en que se encuentren en esa fecha, es decir, su valor de reposición.

- Animales: inventariados por su valor de mercado, que es el precio de mercado existente para el tipo de animal en la zona, descontados los gastos de venta. 
Tabla 1. Total de variables en el estudio.

\begin{tabular}{|c|c|c|}
\hline \multicolumn{2}{|c|}{ Costos Directos } & \multirow{2}{*}{ Gastos generales } \\
\hline Alimentos intrapredial & Otros costos directos & \\
\hline Costo praderas & Productos veterinarios & Combustible y lubricante \\
\hline Concentrado predial & Productos mastitis & Mantención y reparación \\
\hline Leche crianza & Reposición & Electricidad \\
\hline Ensilaje & Lubricantes & Ropa de trabajo \\
\hline Fardos & Productos y equipos ordeña & Comisiones \\
\hline Chancado avena & Detergente alcalino & Contabilidad \\
\hline Otros alimentos & Detergente ácido & Interés financiero \\
\hline Alimentos extrapredial & Higienizantes & Teléfono \\
\hline Concentrado & Utensilios de aseo & Remuneraciones \\
\hline Sustituto lácteo & Análisis de calidad & Asesoría \\
\hline Fardos & Descuento centro acopio lechero (CAL) & Otros (servicios, impuestos, \\
\hline Melaza & Honorarios profesionales & contribuciones y seguros) \\
\hline Chancado avena & Remuneraciones & \\
\hline Maíz & Inseminación & \\
\hline Afrecho raps & Combustibles & \\
\hline Sales minerales & Otros costos & \\
\hline
\end{tabular}

\section{Resultados y Discusión}

\section{Costos totales de los agricultores por método}

En general los costos calculados por el método de diferencia residual son más altos que aquellos calculados por el método de producción equivalente (Tabla 2).

El promedio del costo total para los 14 agricultores calculado por el MCDR fue de \$100,5, mientras que para el MCPE solo alcanzó a \$ 89,77 (diferencia de \$10,73).

Solo cinco agricultores presentaron mayores costos por el MCPE, pero en dichos casos las diferencias con el cálculo hecho por el método de diferencia residual fueron pequeñas.

\section{Análisis estadísticos aplicados a los métodos de costeo por diferencia residual (MCDR) y por producción equivalente (MCPE)}

Debido a la presencia de una cantidad importante de valores mínimos (cercanos a cero) no se consideraron algunas variables de escasa frecuencia para efectos del análisis estadístico. Estas fueron afrecho raps y maíz extrapredial, que se presentaron en solo un caso. Dentro de la producción intrapredial no se consideraron las variables concentrado predial, chancado de avena, diésel y otros alimentos.

Análisis de cluster jerárquico. El análisis por cluster jerárquico para el MCDR genera cuatro grupos. El grupo A se compone de casi un tercio de los casos en estudio y es el que tiene la mayor superficie destinada al rubro lechería. El grupo B se compone de la mitad de los casos, con antecedentes que se encuentran cercanos a la media, con los mejores precios pagados por litro de leche y con los costos más bajos de producción. El grupo $\mathrm{C}$ se compone de un caso y sus antecedentes se encuentran fuera de la media, con precios pagados por litro de leche similares a la media y con los costos más altos de producción. El grupo D se sumó a los antecedentes del grupo $\mathrm{C}$ debido a que está formado por solo un caso. Mayores detalles se pueden revisar en el Tabla 4.

Para el caso del MCPE, el grupo A se compone de cinco casos, unidos por alta similitud. Los activos totales se encuentran por sobre la media, los indicadores de productividad son bajos y las variables de costo praderas, concentrados y productos veterinarios son bajos. Es el grupo que tiene los menores costos de producción. El grupo B se compone de cinco casos, donde el precio promedio pagado al productor de $\$ 92,8 / \mathrm{L}$, con un costo total según el método de $\$ 70,6 / \mathrm{L}$. El grupo C se compone de un caso muy aislado y el precio promedio pagado al productor es de $\$ 97,2 / \mathrm{L}$ y el costo total según el método es de $\$ 126,2 / \mathrm{L}$. El grupo D se compone de un caso, con un precio promedio pagado al productor de $\$ 100,3 / \mathrm{L}$ y un costo total según el método de $\$ 115,6 / \mathrm{L}$. Al igual que en el MCDR, para el caso del MCPE. Hay mayores detalles en el Tabla 3. 
Tabla 2. Resultados de costos por ambos métodos.

\begin{tabular}{lccrrr}
\hline \multirow{2}{*}{ Casos } & \multicolumn{2}{c}{ Variables con valor } & \multicolumn{2}{c}{ Costo total (\$/litro) } & Diferencia \\
\cline { 2 - 5 } & (Número) & $(\%)$ & MCDR & MCPE & $-4,31$ \\
\hline Agricultor 1 & 27 & 58,69 & 105,21 & 100,89 & $-22,58$ \\
Agricultor 2 & 23 & 50,00 & 96,92 & 74,34 & $-9,12$ \\
Agricultor 3 & 32 & 69,56 & 105,08 & 95,96 & $-13,91$ \\
Agricultor 4 & 34 & 73,91 & 108,78 & 94,86 & 4,96 \\
Agricultor 5 & 34 & 73,91 & 80,10 & 85,06 & 7,07 \\
Agricultor 6 & 30 & 65,21 & 99,96 & 107,02 & $-76,47$ \\
Agricultor 7 & 24 & 52,17 & 148,45 & 71,99 & $-2,36$ \\
Agricultor 8 & 37 & 80,43 & 110,10 & 107,75 & $-6,73$ \\
Agricultor 9 & 28 & 60,86 & 132,93 & 126,20 & $-28,07$ \\
Agricultor 10 & 30 & 65,21 & 143,62 & 58,56 & $-5,71$ \\
Agricultor 11 & 33 & 71,73 & 63,85 & 73,96 & 2,51 \\
Agricultor 12 & 23 & 50,00 & 71,44 & 78,67 & 1,37 \\
Agricultor 13 & 27 & 58,69 & 77,30 & 66,38 & 3,06 \\
Agricultor 14 & 26 & 56,52 & 63,32 & 89,77 & $-10,73$ \\
Promedio & & & 100,50 & 20,18 & \\
Desviación estándar & & & 27,67 & \\
\hline
\end{tabular}

MCDR, Método de costeo por diferencia residual; MCPE, Método de costeo por producción equivalente.

Diferencia $=$ MCPE - MCDR.

Tabla 3. Valores promedios del análisis cluster de los grupos por método de diferencia residual (CMDR) y por producción equivalente (MCPE).

\begin{tabular}{|c|c|c|c|c|c|}
\hline \multirow{2}{*}{ Variables } & \multicolumn{4}{|c|}{ Conglomerados } & \multirow{2}{*}{ Promedio } \\
\hline & A & B & C & D & \\
\hline \multicolumn{6}{|l|}{ MCDR } \\
\hline Total activos $(\$)$ & $16.738 .094,8$ & $16.763 .349,6$ & $19.556 .010,7$ & & $17.354 .561,3$ \\
\hline Superficie lechería (ha) & 54,3 & 35,5 & 15,8 & & 36,6 \\
\hline Vaca masa $\left(\mathrm{n}^{\circ}\right)$ & 38,8 & 31,1 & 10,3 & & 28,9 \\
\hline Litros leche /vaca & $3.594,2$ & $2.928,4$ & $2.187,1$ & & $2.959,8$ \\
\hline Producción leche/ha (L) & $2.772,7$ & $2.710,9$ & $1.382,2$ & & $2.443,8$ \\
\hline Costo praderas $(\$ / L)$ & 32,4 & 12,7 & 44,7 & & 25,2 \\
\hline Concentrado $(\$ / \mathrm{L})$ & 5,5 & 4,2 & 1,2 & & 3,9 \\
\hline Productos veterinarios $(\$ / L)$ & 3,5 & 1,9 & 1,4 & & 2,2 \\
\hline Reposición $(\$ / L)$ & 6,6 & 3,4 & 4,3 & & 4,5 \\
\hline Precio promedio pagado $(\$ / \mathrm{L})$ & 106,2 & 95,6 & 97,6 & & 99,1 \\
\hline Costo total $(\$ / \mathrm{L})$ & 106,0 & 79,7 & 141,7 & & 100,5 \\
\hline \multicolumn{6}{|l|}{ MCPE } \\
\hline Total activos $(\$)$ & $20.814 .476,8$ & $17.935 .627,5$ & $5.343 .232,0$ & $5.120 .000,0$ & $17.354 .561,3$ \\
\hline Superficie lechería (ha) & 51,7 & 28,9 & 12,0 & 17,3 & 36,6 \\
\hline Vaca masa $\left(n^{\circ}\right)$ & 40,7 & 24,0 & 8,0 & 8,0 & 28,9 \\
\hline Litros leche/vaca (L/vaca) & $3.538,9$ & $2.503,4$ & $2.944,9$ & $2.238,4$ & $2.959,8$ \\
\hline Producción leche/ha (L/ha) & $2.937,3$ & $2.265,3$ & $1.963,3$ & $1.035,1$ & $2.443,8$ \\
\hline Costo praderas $(\$ / L)$ & 26,1 & 12,4 & 40,9 & 39,5 & 22,2 \\
\hline Concentrado $(\$ / \mathrm{L})$ & 5,3 & 3,2 & 3,2 & 0,2 & 3,9 \\
\hline Productos veterinarios $(\$ / \mathrm{L})$ & 3,0 & 1,5 & 2,1 & 0,7 & 2,1 \\
\hline Reposición (\$/L) & 8,2 & 0,0 & 6,6 & 4,9 & 4,3 \\
\hline Precio promedio pagado $(\$ / \mathrm{L})$ & 105,5 & 92,8 & 97,2 & 100,3 & 99,1 \\
\hline Costo total $(\$ / L)$ & 98,6 & 70,6 & 126,2 & 115,6 & 89,8 \\
\hline
\end{tabular}

MCDR, Método de costeo por diferencia residual; MCPE, Método de costeo por producción equivalente. 
Análisis K-means. Este análisis entrega un resultado donde se reconstruyen los grupos, intentando mover los elementos o casos de un grupo a otro para conseguir un máximo número de variables, con el fin de obtener diferencias significativas entre los conglomerados.

Para el cálculo de costos mediante el MCDR el análisis de K-means entrega tres grupos, cuyos detalles se encuentran en el Tabla 4, al igual que la reagrupación de los casos MCPE. Al comparar las agrupaciones de los métodos de costo se observa que el grupo A no comparte los casos entre métodos. Tienen como consecuencia para el grupo A una disminución de los activos totales en \$21.654.016, un aumento en la superficie de lechería de 2,3 ha, una disminución en la masa de vacas de 3,5 cabezas, un aumento en el rendimiento por vaca de 76,9 L y una disminución por hectárea en \$520,7/L. Los costos de praderas disminuyen $\$ 2,9$, el costo por concentrado disminuye en $\$ 1,5$, los productos veterinarios disminuyen en $\$ 0,86$, el precio promedio pagado por $\mathrm{L}$ de leche disminuye en $\$ 4,0$ y el costo por $L$ aumenta en $\$ 25,1$. En el grupo B se aprecia un aumento de los activos totales en \$ 3.080.039, una disminución en la superficie de lechería de 3,75 ha, una disminución en la masa de vacas de 4,0 cabezas, una disminución en el rendimiento por vaca de $\$ 193,12 /$ L y una disminución por hectárea de $\$ 214,2 / \mathrm{L}$. Los costos de praderas disminuyen en $\$ 1$, el costo por concentrado disminuye en $\$ 0,5$, el costo en productos veterinarios disminuye $\$ 0,2$, el precio promedio pagado disminuye $\$ 1,4$ y el costo por litro disminuye en $\$ 6,7$. En el grupo $C$ hay una disminución de los activos totales en $\$ 10.437 .362$, una disminución en la superficie de lechería de 43,83 ha, una disminución en la masa de vacas de 30,3 cabezas, una disminución en el rendimiento por vaca de $\$ 319,6 / \mathrm{L}$ y una disminución por hectárea de $\$ 259,7 / \mathrm{L}$. Los costos de praderas aumenta $\$ 2,41$, el costo por concentrado disminuye en $\$ 1,9$, en productos veterinarios aumenta en $\$ 0,4$. El precio promedio pagado por $\mathrm{L}$ de leche disminuye $\$ 8,8$ y el costo por L aumenta en $\$ 10.6$. El grupo D no existe en el MCDR, por lo que no se hace una comparación.

De acuerdo con el análisis de ANOVA, y utilizando las pruebas $\mathrm{F}$ solo con una finalidad descriptiva, se tiene que las variables más importantes,

Tabla 4. Valores promedio del análisis K-means de los grupos, por método de costeo.

\begin{tabular}{|c|c|c|c|c|c|}
\hline Variables & A & $\mathrm{B}$ & $\mathrm{C}$ & $\mathrm{D}$ & Media \\
\hline \multicolumn{6}{|l|}{ CMDR } \\
\hline Total activos $(\$)$ & $26.774 .016,0$ & $15.786 .680,9$ & $15.780 .594,8$ & & $17.354 .561,3$ \\
\hline Superficie lechería (ha) & 15,0 & 32,4 & 55,8 & & 36,6 \\
\hline Vaca masa $\left(n^{\circ}\right)$ & 11,5 & 28,5 & 38,3 & & 28,9 \\
\hline Litros por vaca (L/vaca) & $2.161,5$ & $3.007,0$ & $3.264,5$ & & $2.959,8$ \\
\hline Producción leche/ha (L/ha) & $1.555,8$ & $2.776,3$ & $2.223,0$ & & $2.443,8$ \\
\hline Costo praderas $(\$ / L)$ & 42,4 & 14,2 & 38,5 & & 25,2 \\
\hline Concentrado $(\$ / L)$ & 1,7 & 3,9 & 5,2 & & 3,9 \\
\hline Productos veterinarios $(\$ / L)$ & 1,6 & 2,3 & 2,5 & & 2,2 \\
\hline Reposición (\$/L) & 3,5 & 4,0 & 6,2 & & 4,5 \\
\hline Precio promedio pagado $(\$ / \mathrm{L})$ & 96,3 & 96,3 & 105,9 & & 99,1 \\
\hline Costo por litro $(\$ / L)$ & 140,7 & 82,9 & 115,6 & & 100,5 \\
\hline \multicolumn{6}{|l|}{ CMPE } \\
\hline Total activos $(\$)$ & 5.120 .000 & 18.866 .721 & 5.343 .232 & 20.391 .715 & 17.354 .561 \\
\hline Superficie lechería (ha) & 17 & 28,7 & 12,0 & 63,5 & 36,6 \\
\hline $\operatorname{Vaca} \operatorname{masa}\left(\mathrm{n}^{\circ}\right)$ & 8 & 24,5 & 8,0 & 48,0 & 28,9 \\
\hline Litros leche/vaca (L/vaca) & 2.238 & $2.813,9$ & $2.944,9$ & $3.435,6$ & $2.959,8$ \\
\hline Producción leche/ha (L/ha) & 1.035 & $2.562,1$ & $1.963,3$ & $2.679,7$ & $2.443,8$ \\
\hline Costo praderas $(\$ / L)$ & 39 & 13,2 & 40,9 & 31,3 & 22,2 \\
\hline Concentrado $(\$ / \mathrm{L})$ & 0 & 3,4 & 3,2 & 5,9 & 3,9 \\
\hline Productos veterinarios $(\$ / L)$ & 0 & 2,1 & 2,1 & 2,5 & 2,1 \\
\hline Reposición (\$/L) & 4 & 2,4 & 6,6 & 7,6 & 4,3 \\
\hline Precio promedio pagado $(\$ / \mathrm{L})$ & 100 & 95,0 & 97,2 & 107,5 & 99,1 \\
\hline Costo por litro $(\$ / L)$ & 115 & 76,2 & 126,2 & 101,4 & 89,8 \\
\hline
\end{tabular}

MCDR, Método de costeo por diferencia residual; MCPE, Método de costeo por producción equivalente. 
a un nivel de significación del 1 al 5\% para el MCDR, son: Leche crianza, Costo praderas, Inseminación, Fardos intrapredial, Honorarios profesionales, Mantención y reparación, Análisis de calidad, y Otros costos directos. Para el MCPE, además de las variables antes mencionadas, se agregan: Contabilidad, Asesoría, Ensilaje, Comisiones, Fardos extrapredial, Sales minerales, Remuneraciones generales, y Remuneraciones directas.

Análisis discriminante. Este análisis permite la validación estadística con fines de explicación y evaluación de los resultados obtenidos por los análisis anteriores.

En la Tabla 5 se muestran los autovalores, el que mide las desviaciones dentro de los grupos. Se interpreta como la parte de la variabilidad total de la nube de puntos, proyectada sobre el conjunto de todas las funciones atribuibles a la función. Cuando un autovalor es grande, implica que la suma de cuadrados entre grupos es mayor comparado con la suma de cuadrados intragrupos, lo que significa que la función estaría discriminando los grupos (Pérez, 2001).

Para el MCDR se observa que la primera función discriminante refleja la variabilidad del modelo en $66,74 \%$, mientras que la segunda función lo hace en $33,26 \%$, acumulando $100 \%$ entre ambos. Respecto de la misma tabla, la correlación canónica expresa que la primera función discrimina más que la segunda. En el caso del MCPE se observa que la primera función discriminante refleja la variabilidad del modelo en $85,05 \%$, mientras que la segunda función lo hace en $11,55 \%$, acumulando $96,6 \%$ de variabilidad entre ambos. La tercera función solo presenta el 3,39\%, con lo que se completa el $100 \%$, la correlación canónica expresa que la primera función discrimina más que la segunda y la segunda más que la tercera. Sin embargo, para la clasificación se aplicaron las dos primeras funciones.

Al observar las cifras de autovalores se distingue que la primera función del MCDR expresa el menor valor $(5,8)$. Esto no quiere decir que sea el peor, sino que teniendo la capacidad de discriminar, discrimina mucho menos que la primera función del MCPE, que expresa un valor mayor $(267,64)$. Respecto de las varianzas, el MCDR expresa la totalidad de la varianza al nivel de la segunda función. El MCPE lo hace a nivel de la tercera función. Se observa que la primera función tiene el valor más alto de varianza, lo que significa que es capaz de hacer la mayor discriminación entre tres.

Las correlaciones canónicas miden las desviaciones de las puntuaciones discriminantes entre grupos, respecto de las desviaciones totales, sin distinguir grupos. Si su valor es alto (próximo a 1), significa que la dispersión se deberá a las diferencias entre grupos. La correlación canónica para el MCDR tiene los valores más bajos para las dos primeras funciones, explicando que la dispersión se debe a las diferencias entre grupos. Para el MCPE sucede lo mismo para las dos primeras funciones, pero con valores más altos, que explican la dispersión entre grupos. La tercera función es de menor valor, pero suficiente para explicar la dispersión a las diferencias entre grupos.

La lambda de Wilks expresa cuál es la proporción de varianza total de las puntuaciones discriminantes, que no está explicada por las diferencias entre grupos (Tabla 6). El contraste de $\chi^{2}$ asociado a la lambda de Wilks plantea la hipótesis nula de que no existe diferencia en las puntuaciones de las variables independientes entre los grupos. Respecto de lo anterior, el p-value $(\mathrm{P}=0,000)$ del MCDR y MCPE indica que se rechaza la hipótesis nula y se acepta la diferencia entre grupos.

Tabla 5. Autovalores del análisis discriminate por método de costeo.

\begin{tabular}{ccccc}
\hline Función & Autovalor & \% de varianza & \% acumulado & Correlación canónica \\
\hline & & MCDR & & 0,92 \\
2 & 5,80 & 66,74 & 66,74 & 0,86 \\
& 2,89 & 33,26 & 100,00 & 0,998 \\
1 & & MCPE & 85,050 & 0,987 \\
3 & 267,641 & 85,050 & 96,603 & 0,956 \\
\hline
\end{tabular}

MCDR, Método de costeo por diferencia residual; MCPE, Método de costeo por producción equivalente. 
Tabla 6. Valores lambda de Wilks, según método de costeo.

\begin{tabular}{|c|c|c|c|c|}
\hline $\begin{array}{l}\text { Contraste de las } \\
\text { funciones }\end{array}$ & Lambda de Wilks & Chi-cuadrado $\left(\chi^{2}\right)$ & $\mathrm{gl}$ & Significación \\
\hline & & MCDR & & \\
\hline 1 a la 2 & 0,038 & 34,402 & 4 & 0,0000 \\
\hline \multirow[t]{2}{*}{2} & 0,257 & 14,270 & 1 & 0,0002 \\
\hline & & MCPE & & \\
\hline 1 a la 3 & 0,000 & 93,38 & 18 & 0,0000 \\
\hline 2 a la 3 & 0,002 & 48,63 & 10 & 0,0000 \\
\hline 3 & 0,086 & 19,67 & 4 & 0,0006 \\
\hline
\end{tabular}

MCDR, Método de costeo por diferencia residual; MCPE, Método de costeo por producción equivalente.

$\chi_{(15 ; 0,05)}^{2}(\mathrm{P}<0,01)$ altamente significativa.

\section{Conclusiones}

$\mathrm{Al}$ analizar los costos estimados por ambas metodologías el método por diferencia residual mostró valores de costos más altos que el método por producción equivalente.

En el análisis cluster jerárquico se aprecia que ambos métodos conforman grupos diferentes, tres para el método por diferencia residual y cuatro para el método por producción equivalente, haciendo un corte a un mismo nivel.
En el análisis K-means el nivel de significación del 1 al 5\% indica que hay diferencias en el número de variables, ocho para el método por diferencia residual y catorce para el método de producción equivalente.

Los resultados de la aplicación del análisis discriminante muestran que ambos métodos de costos son capaces de discriminar y separar los grupos con una alta significancia para valores $\chi^{2}(\mathrm{P}<0,01)$.

Como este análisis hace la validación estadística, se concluye que no habría diferencia estadística entre ambos métodos de costos por litro de leche.

\section{Literatura Citada}

Baker, N.; Jacobsen, L.; Ramírez, D.

1992. Contabilidad de Costos. $2^{\mathrm{a}}$ ed. México. McGraw-Hill. $743 \mathrm{pp}$.

Díaz de Rada, V.

1998. Diseño de tipologías de consumidores mediante la utilización conjunta del análisis cluster y otras técnicas multivariantes. Revista española de economía agraria, 182: 71-105

Frank, G.

1998a. Cost of production versus cost of production. <http:// cdp.wisc.edu> (4 sept 2002).

Frank, G.

1998b. Calculating your milk production cost and using the results to manage your expenses. $<$ http:// cdp.wisc.edu $>$ (4 sept 2002).

Gondar, J.

2003. Técnicas estadísticas aplicadas a las ciencias sociales. Madrid, España. Data Mining Institute. 320 pp.

Hair, J.; Anderson, R.; Thatham, R.; Black, W.

1999. Análisis multivariante. $5^{\mathrm{a}}$ ed. Madrid, España. PrenticeHall Iberia. 832 pp.

Horngren, C.; Foster, G.; Datar, S.

1996. Contabilidad de costos un enfoque gerencial. $8^{\circ}$ ed. México. Pearson educación. 970 pp.
Horngren, C.; Sundem, G.Y.; Stratton, W.

2001. Introducción a la contabilidad administrativa. $11^{\mathrm{a}} \mathrm{ed}$. México. Pearson educación. 664 pp.

Johnsons, D.

2000. Métodos multivariados aplicados al análisis de datos. México, México. Thomson. 566 pp.

Lerdon, J.; Jara, G.; Parra, N.

1984. Costos de producción del litro de leche e indicadores económicos del rubro lechería en diez predios del país. Universidad Austral de Chile. Valdivia. 48 pp.

Lévy, J.

2003. Análisis multivariable para las ciencias sociales. Madrid, España. Pearsons. 896 pp.

Leyton, J.; Niño, J.

1994. Precios de transferencia y asignación de costos. In XVII jornada de contabilidade, economía e adminitraçao do cone sul. Santos, Brasil. pp. 229-319.

Mallo, C.; Kaplan, R.; Meljem, S.; Giménez C.

2000. Contabilidad de costos y estrategia de gestión. México. Pearson educación. 768 pp.

Pérez, C.

2001. Técnicas estadísticas con SPSS. Madrid, España. Pearson. 592 pp. 